\title{
More Colourful Simplices
}

\author{
Antoine Deza · Tamon Stephen · Feng Xie
}

Received: 14 January 2010 / Revised: 31 May 2010 / Accepted: 25 August 2010 /

Published online: 11 September 2010

(C) Springer Science+Business Media, LLC 2010

\begin{abstract}
We show that any point in the convex hull of each of $(d+1)$ sets of $(d+1)$ points in general position in $\mathbb{R}^{d}$ is contained in at least $\left\lceil(d+1)^{2} / 2\right\rceil$ simplices with one vertex from each set. This improves the known lower bounds for all $d \geq 4$.

Keywords Colourful simplicial depth · Colourful Carathéodory theorem · Discrete geometry
\end{abstract}

\section{Introduction}

A point $p \in R^{d}$ has simplicial depth $k$ relative to a set $S$ if it is contained in $k$ closed simplices generated by $(d+1)$ sets of $S$. This was introduced by Liu [8] as a statistical measure of how representative $p$ is of $S$, and is a source of challenging problems in computational geometry-see for instance [6]. More generally, we consider colourful simplicial depth, where the single set $S$ is replaced by $(d+1)$ sets, or colours, $\mathbf{S}_{1}, \ldots, \mathbf{S}_{d+1}$, and the colourful simplices containing $p$ are generated by taking one point from each set.

Assuming that the convex hulls of the $\mathbf{S}_{i}$ 's contain $p$ in their interior, Bárány's Colourful Carathéodory Theorem [2] shows that $p$ must be contained in some colour-

\footnotetext{
A. Deza $(\bowtie) \cdot$ F. Xie

Advanced Optimization Laboratory, Department of Computing and Software, McMaster University, Hamilton, Ontario, Canada L8S 4K1

e-mail: deza@mcmaster.ca

F. Xie

e-mail: xief@mcmaster.ca

T. Stephen

Department of Mathematics, Simon Fraser University, 8888 University Drive, Burnaby, British

Columbia, Canada V5A 1S6

e-mail: tamon@sfu.ca 
ful simplex. We are interested in determining the minimum number of colourful simplices that can contain $p$ for sets satisfying these conditions. That is, we would like to determine $\mu(d)$, the minimum number of colourful simplices drawn from $\mathbf{S}_{1}, \ldots, \mathbf{S}_{d+1}$ that contain $p \in R^{d}$ given that $p \in \operatorname{int}\left(\operatorname{conv}\left(\mathbf{S}_{i}\right)\right)$ for each $i$. Without loss of generality, we assume that the points in $\bigcup_{i} \mathbf{S}_{i} \cup\{p\}$ are in general position. Besides intrinsic appeal, $\mu(d)$ represents the minimum number of solutions to the colourful linear programming feasibility problem proposed in [4] and discussed in [5].

The quantity $\mu(d)$ was investigated in [5], where it is shown that $2 d \leq \mu(d) \leq$ $d^{2}+1$, that $\mu(d)$ is even for odd $d$, and that $\mu(2)=5$. This paper also conjectures that $\mu(d)=d^{2}+1$ for all $d \geq 1$. Subsequently, [3] verified the conjecture for $d=$ 3 and provided a lower bound of $\mu(d) \geq \max \left(3 d,\left\lceil\frac{d(d+1)}{5}\right\rceil\right)$ for $d \geq 3$, while [9] independently provided a lower bound of $\mu(d) \geq\left\lfloor\frac{(d+2)^{2}}{4}\right\rfloor$. In this note we show:

Theorem 1 For $d \geq 1$, we have $\mu(d) \geq\left\lceil\frac{(d+1)^{2}}{2}\right\rceil$.

This strengthens the previously known lower bound for all $d \geq 4$.

\section{Preliminaries}

Without loss of generality we can take $p=\mathbf{0}$. The sets $\mathbf{S}_{1}, \ldots, \mathbf{S}_{d+1}$ must each contain at least $(d+1)$ points for $\mathbf{0}$ to be in the interior of their convex hulls, and since we are minimizing we can assume they contain no additional points, i.e. that $\left|\mathbf{S}_{i}\right|=d+1$ for each $i$. We assume that all points are distinct, so no point occurs in two $\mathbf{S}_{i}$ 's, and $\mathbf{0}$ is not in any $\mathbf{S}_{i}$. We can scale the points of the $\mathbf{S}_{i}$ 's so that they lie on the unit sphere $\mathbb{S}^{d-1}: \mathbf{0}$ is in a simplex after scaling if and only if it was in the simplex before scaling.

We call a set of points drawn from the $\mathbf{S}_{i}$ 's colourful if it contains at most one point from each $\mathbf{S}_{i}$. We call a colourful set of $d$ points which misses $\mathbf{S}_{i}$ an $\widehat{i}$-transversal. Note that $\hat{i}$-transversals generate full dimensional pointed colourful cones; we will say that a transversal spans a point if the point is contained in the associated cone. A key observation is that colourful simplices containing $\mathbf{0}$ are generated whenever the antipode of a point of colour $i$ is spanned by an $\widehat{i}$-transversal.

In particular, we look at the combinatorial octahedra, or cross polytopes, generated by pairs of disjoint $\hat{i}$-transversals. We rely on the topological fact that every octahedron $\Omega$ either covers all of $\mathbb{S}^{d-1}$ with colourful cones, or, every point $x \in \mathbb{S}^{d-1}$ that is covered by colourful cones from $\Omega$ is covered by at least two distinct such cones. In the case where the points of $\Omega$ form an octahedron in the geometric sense, these correspond to the cases where $\mathbf{0}$ is inside and outside $\Omega$ respectively. For a proof, see for example the Octahedron Lemma of [3]. We remark that a given octahedron contains $2^{d}$ transversals, though we specify only two disjoint ones to generate it.

Our strategy for finding distinct colourful simplices is to begin with a transversal that generates at least one colourful simplex, and get further points from octahedra that include this transversal. We will break into cases based on the number of 
colourful simplices generated by the initial transversal and how many of the octahedra cover $\mathbb{S}^{d-1}$.

\section{Proof of the Theorem 1}

We know that at least one colourful simplex contains $\mathbf{0}$. Therefore we have an antipode of colour $(d+1)$ lying in the cone generated by a $\widehat{d+1}$-transversal $T$. Without loss of generality we can number the points of $\mathbf{S}_{1}, \mathbf{S}_{2}, \ldots, \mathbf{S}_{d}$ so that point $(d+1)$ of $S_{i}$ is included in $T$. The remaining points of the $S_{i}$ 's can be numbered arbitrarily. Let $T_{i}$ be the set that contains the points numbered $i$ from $\mathbf{S}_{1}, \mathbf{S}_{2}, \ldots, \mathbf{S}_{d+1}$. Then each $T_{i}$ is a $\widehat{d+1}$-transversal and $T_{d+1}=T$. Further, the sets $T_{1}, T_{2}, \ldots, T_{d+1}$ are pairwise disjoint. Let $L$ be the set of antipodes of colour $(d+1)$ spanned by $T_{d+1}$, where $|L|=l>0$.

\subsection{Points from $d$ Octahedra that Share a Transversal}

Now consider the $d$ octahedra $\Omega_{1}, \Omega_{2}, \ldots, \Omega_{d}$ given by pairing $T_{i}$ with $T_{d+1}$ for $i=$ $1,2, \ldots, d$. Except for the common transversal $T_{d+1}$, every $\widehat{d+1}$-transversal found among the $\Omega_{i}$ 's is distinct. For each $i, \Omega_{i}$ may or may not cover all of $\mathbb{S}^{d-1}$. Suppose that $b$ of the octahedra cover $\mathbb{S}^{d-1}$. There are $(d+1-l)$ antipodes of colour $(d+1)$ that are not spanned by $T_{d+1}$, and hence must be spanned by a different transversal from each of these octahedra. This gives us a total of $b(d+1-l)$ distinct simplices containing $\mathbf{0}$. Now there remain $(d-b)$ octahedra that do not span all of $\mathbb{S}^{d-1}$. By the Octahedron Lemma, each of the $l$ antipodes spanned by $T_{d+1}$ must also be spanned by a second transversal from the octahedron generated by $T_{d+1}$ and $T_{i}$. So we find an additional $(d-b) l$ distinct simplices along with the $l$ simplices generated by the antipodes with $T_{d+1}$ itself. This brings us to a total of $l+b(d+1-l)+(d-b) l=$ $(d+1)(b+l)-2 b l$ distinct colourful simplices containing $\mathbf{0}$ through this simple argument.

\subsection{Choice of $T_{d+1}$}

In the above argument, $T_{d+1}$ can be any $\widehat{d+1}$-transversal containing an antipode of colour $(d+1)$. In the construction of previous lower bounds, it was noted that if $\operatorname{depth}(\mathbf{0})$ is low, then there must be a portion of $\mathbb{S}^{d}$ lightly covered by colourful cones. That is to say, if each antipode of colour $(d+1)$ is spanned by at least $j$ $\widehat{d+1}$-transversals, then $\operatorname{depth}(\mathbf{0}) \geq j(d+1)$. We can take $T_{d+1}$ to be a transversal spanning the least covered antipode. As we move through the possible values of $i$ in the argument of Sect. 3.1, whenever the octahedron fails to cover $\mathbb{S}^{d-1}$ we will see a new cone covering the lightly covered antipode. Hence $(j-1)+b \geq d$. We thus have that $\operatorname{depth}(\mathbf{0})$ is at least $\max [j(d+1),(d+1)(b+l)-2 b l]$ with $j \geq 1,1 \leq b, l \leq d$, and $j+b \geq d+1$.

As long as $l \leq \frac{d+1}{2}$, this gives the desired result: by taking either $j \geq \frac{d+1}{2}$ or $b \geq \frac{d+2}{2}$ we get $\operatorname{depth}(\mathbf{0}) \geq \frac{d^{2}+2 d+1}{2}$. 


\subsection{Single Transversals Spanning Many Antipodes}

This leaves only the case where $\ell \geq \frac{d+2}{2}$. In this situation, we begin with $l$ simplices containing $\mathbf{0}$ differing only in the $(d+1)$ st colour. We can repeat this exercise for each colour, in which case we will either find that for each colour $i, l_{i} \geq \frac{d+2}{2}$, or, for some colour $i, l_{i} \leq \frac{d+1}{2}$. In the latter case, we apply the analysis above to get at least $\frac{d^{2}+2 d+1}{2}$ distinct simplices containing zero.

If it happens that we get $l_{i} \geq \frac{d+2}{2}$ for each $i$, then for each $i$ we have a set $L_{i}$ of at least $l_{i}$ antipodes of colour $i$ which lie on a single $\widehat{i}$-transversal $U_{i}$. These generate $(d+1)$ sets $X_{1}, X_{2}, \ldots, X_{d+1}$ of at least $l=\min _{i}\left(l_{i}\right) \geq \frac{d+2}{2}$ colourful simplices. There may be some duplication between sets, but we note that the simplices within each set are distinct and differ only in the $i$ th colour.

We can identify the simplices that make up the $X_{i}$ 's with vectors in $\{1,2, \ldots$, $d+1\}^{d+1}$. We find it helpful to consider them as vectors in $\mathbb{R}^{d+1}$ unrelated to the initial configuration.

A simplex $\alpha_{d}$ belonging to a given $X_{i}$ is represented by a vector in $\mathbb{R}^{d+1}$ in the following way. The axes correspond to the $d+1$ colours, and the $q$ th coordinate is set to the index in $S_{q}$ of the point of colour $q$ of $\alpha_{d}$. We recall that the index of points in $S_{q}$ is set by the arbitrary numbering of points of colour $q$ proposed at the beginning of Sect. 3.

The vectors associated to the simplices from a given $X_{i}$ lie on a line segment in the $i$ th coordinate direction. If a simplex is in both $X_{i}$ and $X_{q}$, then the associated vector must lie at the intersection of the corresponding line segments.

Lemma There are at most $d$ duplicate vectors in the union of the $X_{i}$ 's, where a vector that is in $k+1$ sets is counted as $k$ duplicate vectors.

Proof Consider adding the sets iteratively. We will say that two sets are in the same component if they contain a common point, and extend this to an equivalence relation. We remark that each component is contained in the topological component formed by taking the union of the line segments associated to the $X_{i}$ 's, but a given topological component will contain multiple components if the points of intersection of the line segments are not included in the corresponding $X_{i}$ 's.

We begin with $c=0$ components and $k=0$ duplicate vectors. Each added set either creates a new component or intersects $r$ components, producing $r$ duplicate vectors while reducing the number of components by $(r-1)$ through the equivalence relation. Therefore at each step $c+k$ increases by 1 . Upon termination, we will have at least one component, and hence at most $d$ duplicate vectors.

Then the $X_{i}$ 's contain distinct simplices except possibly for up to $d+1-c \leq d$ repeats arising in this construction, where $c$ is the number of components. This gives us a total of $(d+1) l-(d+1-c)=(d+1)(l-1)+c$ distinct simplices containing $\mathbf{0}$.

However, if $c$ is small, we can readily find additional distinct simplices containing 0 by observing that for a fixed colour $i$, for instance one attaining $l=l_{i}$, we also have $(d+1-l)$ antipodes outside of $L_{i}$. Each of these antipodes must generate some colourful simplex containing $\mathbf{0}$. In fact, for each antipode omitted, we could get $\frac{d+1}{2}$ 
simplices since either $l_{i}$ or $b$ is this large, but it does not improve our worst case. Call this set of simplices $M$, and again consider them as vectors in $\mathbb{R}^{d+1}$. They are not included among the vectors associated to simplices in $X_{i}$, since they have different values of coordinate $i$.

The vectors associated to simplices in $M$ could duplicate vectors from components other than the one containing $X_{i}$. However, each such component has a fixed value of colour $i$. If $c-1 \geq d+1-l$ it may be the case that all such simplices are repeats, but our guarantee is $(d+1)(l-1)+c \geq d l+1$. If $c-1<d+1-l$ we get at least $d+2-l-c$ additional distinct simplices from vertices omitted from the $(d+1)$ sets. This again guarantees us at least $(d+1)(l-1)+c+(d+2-l-c)=d l+1$ distinct simplices.

Now as $l \geq \frac{d+2}{2}$ we get at least $d \frac{d+2}{2}+1=\frac{d^{2}+2 d+2}{2}$ distinct simplices containing $\mathbf{0}$. Thus our overall worst case for this analysis is at $\frac{d^{2}+2 d+1}{2}=\frac{(d+1)^{2}}{2}$, which can be rounded up to an integer when $d$ is even. This improves the known bounds for $d \geq 4$, in particular from 12 to 13 when $d=4$. We remark that unlike previous general approaches, this analysis gives the tight bound of 5 when $d=2$.

\section{A Combinatorial Generalization}

The methods in Sect. 3 rely on the combinatorial structure of the vectors representing the simplices. Indeed, there is a nice generalization of the colourful simplicial depth problem to systems of vectors of in $\{1,2, \ldots, d+1\}^{d+1}$.

Given sets $\mathbf{S}_{1}, \ldots, \mathbf{S}_{d+1}$ as in Sect. 1, we form the system of vectors $\mathbf{V}$ where $\mathbf{v}=\left(s_{1}, \ldots, s_{d+1}\right)$ is in $\mathbf{V}$ exactly if the colourful simplex described by $\mathbf{v}$ contains $\mathbf{0}$. In this context, $\hat{i}$-transversals are simply vectors with the $i$ th coordinate removed, and octahedra are pairs of disjoint $\hat{i}$-transversals. The system $\mathbf{V}$ has the following two properties:

1. Every element of $\{1,2, \ldots, d+1\}^{d+1}$ is in some $\mathbf{v} \in \mathbf{V}$. This is the combinatorial requirement from Bárány's Colourful Carathéodory Theorem.

2. For any octahedron $\varnothing$, the parity of the set of vectors using points from $\varnothing$ and a fixed point $s_{i}$ for the $i$ th coordinate is the same for all choices of $s_{i}$. For a system $\mathbf{V}$ arising from colourful simplices, the parity is odd when the octahedron $\varnothing$ contains $\mathbf{0}$, and even when it does not. This is a purely combinatorial version of the Octahedron Lemma mentioned in Sect. 2.

Question 4.1 For a given $d \geq 2$, what is the size $v(d)$ of a minimal system $\mathbf{V}$ of vectors in $\{1,2, \ldots, d+1\}^{d+1}$ satisfying properties 1 and 2 ?

The system corresponding to the conjectured minimal core colourful Carathéodory configuration from [5] satisfies properties 1 and 2 with $d^{2}+1$ vectors, so $v(d) \leq$ $\mu(d) \leq d^{2}+1$. Clearly $v(d) \geq d+1$. An exhaustive computer search on a laptop shows in a few seconds that $v(2)>4$ and in a few hours that $v(3)>8$. In other words, this approach computationally verifies that $\mu(2)=5$ and $\mu(3)=10$ (using the fact that $\mu$ (3) must be even). 


\section{A Generalized Core}

As a final remark, we mention the recent generalization of the Colourful Carathéodory Theorem in [7] and [1], in which the condition of $\mathbf{0}$ being in the convex hull of each $\mathbf{S}_{i}$ is relaxed to require $\mathbf{0}$ to only be in the convex hull of $\mathbf{S}_{i} \cup \mathbf{S}_{j}$ for each $i \neq j$. It is natural to ask whether the minimum number of colourful simplices containing $\mathbf{0}$ is lower for configurations satisfying these weaker conditions. Call the analogous quantity $\mu^{\diamond}(d)$.

In fact, the construction of [5] can be modified in this to produce configurations showing that $\mu^{\diamond}(d) \leq d+1$ by fixing the points of colours $1,2, \ldots, d$ in the same way and then clustering all antipodes of the final colour in region that is covered by only a single colourful cone from the first $d$ colours. In this case the relaxed conditions are satisfied almost trivially since $\mathbf{0}$ is in $\operatorname{conv}\left(\mathbf{S}_{i}\right)$ for $i=1,2, \ldots, d$. We note that in this configuration, each colour from $1, \ldots, d$ has a unique point which is a generator for all $(d+1)$ colourful simplices colourful simplices containing $\mathbf{0}$. In other words, in contrast to the situation when $\mathbf{0}$ is in all the $\mathbf{S}_{i}$ 's, some (in fact, most) points from the $\mathbf{S}_{i}$ generate no colourful simplices containing $\mathbf{0}$.

The following simple argument shows that $\mu^{\diamond}(2)=3$. Using the assumptions of Sect. 2, we place the points of the first two colours on the unit circle around $\mathbf{0}$. The condition $\mathbf{0} \in \operatorname{conv}\left(\mathbf{S}_{1} \cup \mathbf{S}_{2}\right)$ then means that every half-circle contains a point from $\mathbf{S}_{1} \cup \mathbf{S}_{2}$. If the circle is covered by colourful cones, then each antipode of the remaining colour generates a colourful simplex containing $\mathbf{0}$ and we are done. Otherwise, some segment of the circle is not covered by any colourful cone. This segment must be bounded by two points $p$ and $p^{\prime}$ of the same $\mathbf{S}_{i}$, say $\mathbf{S}_{1}$. The three points of $\mathbf{S}_{2}$ then are on the longer arc between these points, and for each point of $\mathbf{S}_{2}$, every point on the longer arc is covered by a colourful cone using that point and either $p$ or $p^{\prime}$. The condition that $\mathbf{0} \in \operatorname{conv}\left(\mathbf{S}_{2} \cup \mathbf{S}_{3}\right)$ forces at least one of the antipodes of $\mathbf{S}_{3}$ to lie in the arc that spans the three points of $\mathbf{S}_{2}$.

Finally, we remark that we can generalize $\mu^{\diamond}(d)$ combinatorially to $v^{\diamond}(d)$ analogously to Sect. 4. Combinatorial Property 2 must still hold for such configurations, but Property 1 fails in the constructions above. Nevertheless, we can quickly verify computationally the $v^{\diamond}(3)=\mu^{\diamond}(3)=4$.

Acknowledgements This work was supported by grants from the Natural Sciences and Engineering Research Council of Canada (NSERC) and MITACS, and by the Canada Research Chairs program. The authors would like to thank the referees for helpful comments and Imre Bárány for initiating the discussion in Sect. 4.

\section{References}

1. Arocha, J.L., Bárány, I., Bracho, J., Fabila, R., Montejano, L.: Very colorful theorems. Discrete Comput. Geom. 42(2), 142-154 (2009)

2. Bárány, I.: A generalization of Carathéodory's theorem. Discrete Math. 40(2-3), 141-152 (1982)

3. Bárány, I., Matoušek, J.: Quadratically many colorful simplices. SIAM J. Discrete Math. 21(1), 191198 (2007)

4. Bárány, I., Onn, S.: Colourful linear programming and its relatives. Math. Oper. Res. 22(3), 550-567 (1997) 
5. Deza, A., Huang, S., Stephen, T., Terlaky, T.: Colourful simplicial depth. Discrete Comput. Geom. 35(4), 597-604 (2006)

6. Fukuda, K., Rosta, V., Data depth and maximal feasible subsystems. In: Avis, D., Hertz, A., Marcotte, O. (eds.) Graph Theory and Combinatorial Optimization, pp. 37-67. Springer, New York (2005)

7. Holmsen, A.F., Pach, J., Tverberg, H.: Points surrounding the origin. Combinatorica 28(6), 633-644 (2008)

8. Liu, R.Y.: On a notion of data depth based on random simplices. Ann. Stat. 18(1), 405-414 (1990)

9. Stephen, T., Thomas, H.: A quadratic lower bound for colourful simplicial depth. J. Comb. Optim. 16(4), 324-327 (2008) 